Baseline

\title{
A metabolomic study on the biological effects of metal pollutions in oysters Crassostrea sikamea
}

\author{
Chenglong Ji ${ }^{\mathrm{a}}$, Qing Wang ${ }^{\mathrm{a}}$, Huifeng $\mathrm{Wu}^{\mathrm{a}, *}$, Qiaoguo Tan ${ }^{\mathrm{b}}$, Wen-Xiong Wang ${ }^{\mathrm{c}}$

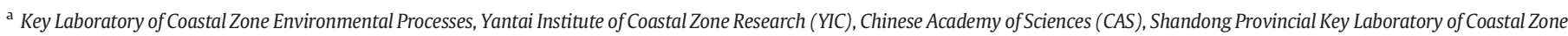 \\ Environmental Processes, YICCAS, Yantai 264003, PR China \\ ${ }^{\mathrm{b}}$ Key Laboratory of the Coastal and Wetland Ecosystems, Ministry of Education, College of Environment and Ecology, Xiamen University, Xiamen 361102, PR China \\ c Division of Life Science, The Hong Kong University of Science and Technology (HKUST), Clearwater Bay, Kowloon, Hong Kong
}

\section{A R T I C L E I N F O}

\section{Article history:}

Received 8 August 2015

Received in revised form 12 November 2015

Accepted 16 November 2015

Available online 23 November 2015

\section{Keywords:}

Metal pollution

Crassostrea sikamea

Metabolomics

${ }^{1}$ H NMR

\begin{abstract}
A B S T R A C T
Metal pollution has become a great threat to organisms in the estuaries in South China. In the present study, the oysters Crassostrea sikamea were collected from one clean (Jiuzhen) and five metal polluted sites (Baijiao, Fugong, Gongqian, Jinshan and Songyu). The tissue metal concentrations in oysters indicated that the five metal sites were polluted by several metals, including $\mathrm{Cr}, \mathrm{Ni}, \mathrm{Co}, \mathrm{Cu}, \mathrm{Zn}, \mathrm{Ag}, \mathrm{Cd}$ and $\mathrm{Pb}$ with different patterns. Especially, $\mathrm{Cu}$ and $\mathrm{Zn}$ were the major contaminants in Baijiao, Fugong and Jinshan sites. The metabolic responses in oysters C. sikamea indicated that the metal pollutions in BJ, FG, JS and SY sites induced disturbances in osmotic regulation and energy metabolism via different metabolic pathways. However, the metal pollution in GQ site mainly influenced the osmotic regulation in the oysters C. sikamea. This study demonstrates that NMR-based metabolomics is useful to characterize metabolic responses induced by metal pollution.
\end{abstract}

(c) 2015 Elsevier Ltd. All rights reserved.
Due to the discharge of industrial effluents, metal pollution has become a serious environmental problem in the estuarine and coastal regions in South China. Tan et al. (2015) found that the two species of oysters, Crassostrea hongkongensis and Crassostrea sikamea, from the metal-polluted sites accumulated extremely high concentrations of $\mathrm{Cu}$ in their tissues, which made C. hongkongensis and C. sikamea blue (light turquoise) and green (asparagus green), respectively. The $\mathrm{Cu}$ concentrations in the metal-contaminated oysters reached as high as $19,000 \mu \mathrm{g} / \mathrm{g}$ dry weight, however, the oysters still can survive and grow up in the metal-contaminated sites along Jiulong River Estuary, Fujian Province, China (Weng and Wang, 2014). Since excessive metals can induce adverse effects in aquatic organisms, the great concern has been raised over the health risks to aquatic organisms (Wang et al., 2014).

The marine filter-feeders, such as mussels, clams, cockles and oysters, can control the abundances of primary producers. Therefore, they can maintain the marine ecosystem health in many estuarine systems (Weng and Wang, 2014). These filter-feeders are also known as environmental biomonitors for metals (Goldberg et al., 1983). Among these filter-feeders, the oysters $C$. hongkongensis and $C$. sikamea are the hyperaccumulators of $\mathrm{Cu}$ and $\mathrm{Zn}$ (Tan et al., 2015). In addition, these oysters are economically important species and widely consumed in South China. Therefore, it is necessary to investigate the biological stress of metal pollution to oysters.

\footnotetext{
* Corresponding author.

E-mail address: hfwu@yic.ac.cn (H. Wu).
}

Although the traditional biochemical indices, such as lysosomal membrane stability and anti-oxidative enzyme activities, are usable to characterize the biological effects induced by metal pollutions, they lack the capability to perform a global test of a certain class of biological molecules that can be filtered for the biomarkers related to biological effects (Regoli, 2000; de Almeida et al., 2004; Rank et al., 2007; Cappello et al., 2013a). With the rapid development of system biology techniques including genomics, transcriptomics, proteomics and metabolomics, researchers can carry out a global analysis on the molecules to obtain the biomarkers related to the biological effects induced by environmental stressors (Ji et al., 2013; Santos et al., 2010; Williams et al., 2009). Among these system biology techniques, metabolomics focuses on all the small molecular metabolites $(<1000 \mathrm{Da})$ that are the end products of metabolism (Jones et al., 2008; Viant et al., 2003). A global analysis on the metabolome profile in a biological system can provide metabolic responses induced by environmental stressors (Fasulo et al., 2012; Kwon et al., 2012; Cappello et al., 2013a, 2013b). And it has been also widely applied in multiple areas such as food quality (Tarachiwin et al., 2008), ecotoxicology (Jones et al., 2008) and plant sciences (Kim et al., 2010). Cappello et al. (2013a, 2013b) identified the metabolic responses $\mathrm{Hg}$ and PAHs in mussel Mytilus galloprovincialis caged in anthropogenic-impacted sites along the Augusta coastline (Sicily, Italy) and confirmed these mussels had suffered from adverse environmental condition. To investigate the biological effects of heavy metal pollution, Kwon et al. (2012) employed NMR-based metabolomics to compare the metabolic profiles in marine mussels (Mytilus edulis) sampled from a metal-polluted area (Onsan Bay) and a clean area 
(Dokdo area). They found that NMR-based metabolomics was applicable to characterize metal pollution-induced biological effects.

In this study, we applied NMR-based metabolomics to investigate metabolic responses in oyster (C. sikamea) to metal pollution. The oysters C. sikamea were collected from six sites (Baijiao, Fugong, Gongqian, Jinshan, Songyu and Jiuzhen) along Jiulong River Estuary. Among these sampling sites, the former five sites were contaminated by different concentrations of metals (such as zinc, copper, manganese and lead etc.), respectively, and Jiuzhen was a relatively clean site used as the reference. Due to the small size of oyster $C$. sikamea, the whole soft tissues of individual oysters were examined for the metabolic profiles. The aims of this study were to characterize the metabolic responses and hence biological effects in oysters $C$. sikamea exposed to metal pollutions using NMR-based metabolomics.

During the low tides, the contaminated oysters $C$. sikamea were collected from Baijiao (BJ, $24^{\circ} 28^{\prime} 2^{\prime \prime} \mathrm{N}, 117^{\circ} 56^{\prime} 19^{\prime \prime} \mathrm{E}$ ), Fugong (FG, $24^{\circ} 22^{\prime}$ $58^{\prime \prime} \mathrm{N}, 117^{\circ} 54^{\prime} 13^{\prime \prime} \mathrm{E}$ ), Gongqian (GQ 24 $24^{\prime} 1^{\prime \prime} \mathrm{N}, 117^{\circ} 58^{\prime} 15^{\prime \prime} \mathrm{E}$ ), Jinshan

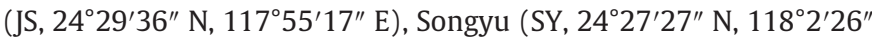
E) sites along the contaminated Jiulong River Estuary, and the control oysters were collected from the harbor of Jiuzhen (JZ, $24^{\circ} 2^{\prime} 38^{\prime \prime} \mathrm{N}$, $117^{\circ} 42^{\prime} 26^{\prime \prime} \mathrm{E}$ ) site from the nearby relatively clean Jiuzhen estuary, Fujian of China, on December 18th, 2012 (Fig. 1). The Jiulong River Estuary is an important area for oyster culture, and the average surface salinity ranged from 14 to 26 psu as for tidal actions (Liu and Wang, 2012). However, evidences confirmed that the Jiulong River Estuary was contaminated by metals (Liu and Wang, 2012). Nine or ten individual oysters with similar sizes were sampled from each site, and the whole soft tissues were immediately dissected and flash frozen in liquid $\mathrm{N}_{2}$. After transported to our laboratory, these oyster samples were stored at $-80^{\circ} \mathrm{C}$ before metabolite extraction and metal analysis. All the practical procedures for oyster sampling were strictly performed according to the guidelines suggested by Hines et al. (2007) and Vidal-Liñán and Bellas (2013).

Polar metabolites were extracted from the oyster tissues by a modified extraction protocol using methanol/chloroform (Zhang et al., 2011). Briefly, the oyster tissue ( $100 \mathrm{mg}$ ) was homogenized using a high throughput homogenizer (Precellys 24, Bertin Technologies, France) and extracted in $4 \mathrm{~mL} / \mathrm{g}$ of methanol, $0.85 \mathrm{~mL} / \mathrm{g}$ of water and $2 \mathrm{~mL} / \mathrm{g}$ of chloroform. The mixture was shaken and centrifuged ( $5 \mathrm{~min}, 3000 \mathrm{~g}$, at $4{ }^{\circ} \mathrm{C}$ ), and the supernatant substance was removed. A total of $2 \mathrm{~mL} / \mathrm{g}$ of chloroform and $2 \mathrm{~mL} / \mathrm{g}$ of water were added to the supernatant, and the mixture was vortexed and then centrifuged again $\left(10 \mathrm{~min}, 3000 \mathrm{~g}, 4^{\circ} \mathrm{C}\right)$. The methanol/water layer with polar metabolites was transferred to a glass vial. The sample was dried in a centrifugal concentrator and stored at $-80^{\circ} \mathrm{C}$. It was subsequently re-suspended in $600 \mu \mathrm{L}$ of $100 \mathrm{mM}$ phosphate buffer $\left(\mathrm{Na}_{2} \mathrm{HPO}_{4}\right.$ and $\mathrm{NaH}_{2} \mathrm{PO}_{4}$ with $0.5 \mathrm{mM}$ TSP, pH 7.0) in $\mathrm{D}_{2} \mathrm{O}$. The mixture was vortexed and then centrifuged at $3000 \mathrm{~g}$ for $5 \mathrm{~min}$ at $4{ }^{\circ} \mathrm{C}$. The supernatant substance $(550 \mu \mathrm{L})$ was then pipetted into a 5 NMR tube for NMR analysis.

Extracts of oyster samples were analyzed on a Bruker AV 500 NMR spectrometer performed at $500.18 \mathrm{MHz}$ (at $298 \mathrm{~K}$ ) (Zhang et al., 2011). One-dimensional (1-D) ${ }^{1} \mathrm{H}$ NMR spectra were obtained using a $11.9 \mu$ s pulse, $6009.6 \mathrm{~Hz}$ spectral width, mixing time $0.1 \mathrm{~s}$, and $3.0 \mathrm{~s}$ relaxation delay with standard 1D NOESY pulse sequence, with 128 transients collected into 16384 data points. Datasets were then zero-filled to 32768 points, and exponential line-broadenings of $0.3 \mathrm{~Hz}$ were applied before Fourier transformation. All ${ }^{1} \mathrm{H}$ NMR spectra were phased, baseline-corrected, and calibrated (TSP at $0.0 \mathrm{ppm}$ ) manually using TopSpin (version 2.1, Bruker).

All one dimensional ${ }^{1} \mathrm{H}$ NMR spectra were converted to a data matrix using the custom-written ProMetab software in Matlab version 7.0 (The MathsWorks, Natick, MA, USA) (Viant et al., 2003). Each spectrum was segmented into bins with a width of $0.005 \mathrm{ppm}$ between 0.2 and $10.0 \mathrm{ppm}$. The bins of residual water peak between 4.70 and $5.20 \mathrm{ppm}$ were excluded from all the NMR spectra. The total spectral area of the remaining bins was normalized to unity to facilitate the comparison between the spectra. All the NMR spectra were generalized log transformed with a transformation parameter $\lambda=2.0 \times 10^{-8}$ to stabilize the variance across the spectral bins and to increase the weightings of the less intense peaks (Zhang et al., 2011). Data were mean-centered before multivariate data analysis.

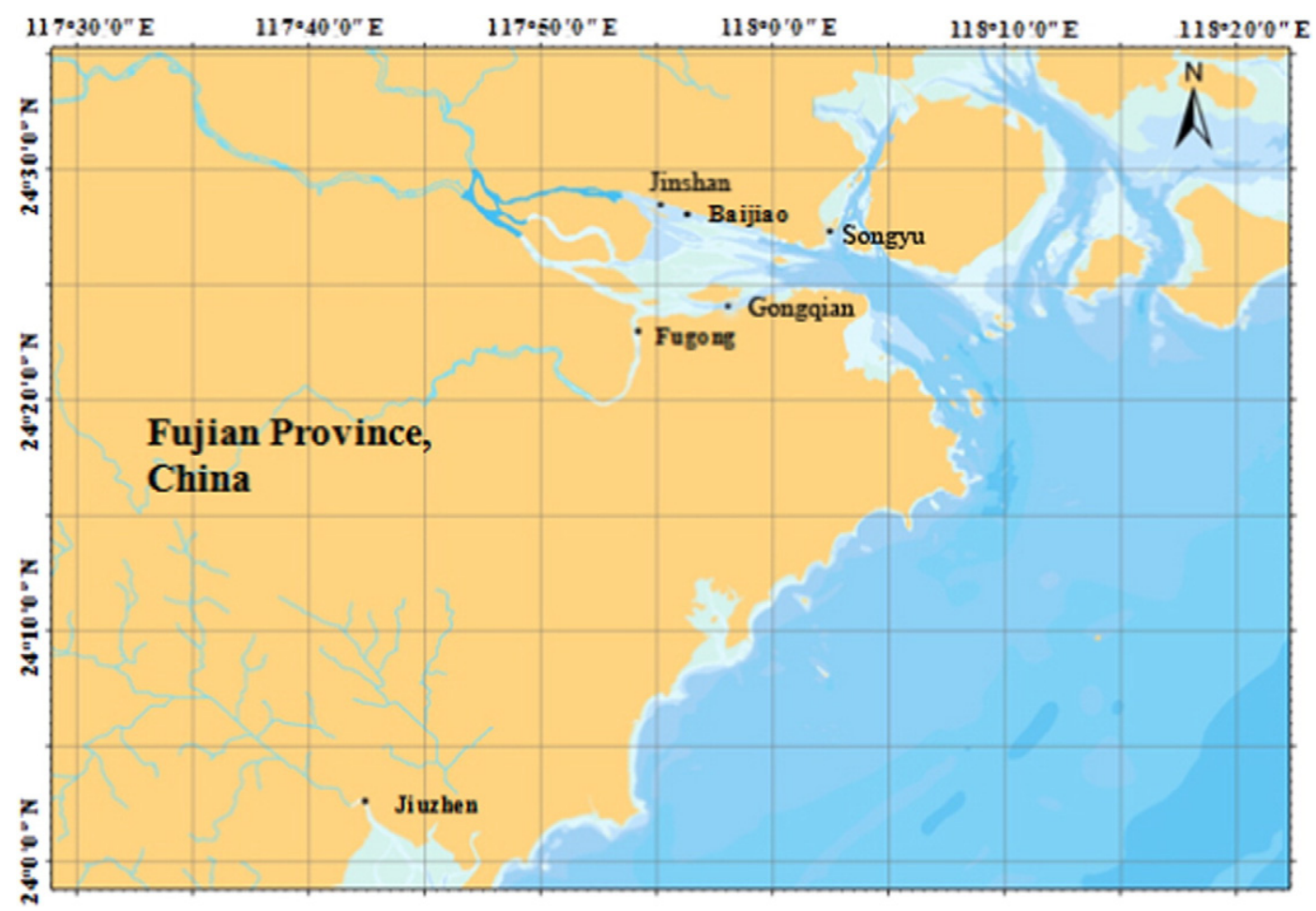

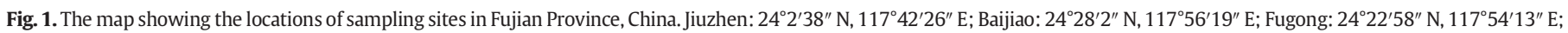
Gongqian: $24^{\circ} 24^{\prime} 1^{\prime \prime} \mathrm{N}, 117^{\circ} 58^{\prime} 15^{\prime \prime}$ E; Jinshan: $24^{\circ} 29^{\prime} 36^{\prime \prime} \mathrm{N}, 117^{\circ} 55^{\prime} 17^{\prime \prime}$ E; Songyu: $24^{\circ} 27^{\prime} 27^{\prime \prime} \mathrm{N}, 118^{\circ} 2^{\prime} 26^{\prime \prime} \mathrm{E}$. 
The unsupervised pattern recognition method, principal component analysis (PCA) was used to reduce the dimensionality of the data and separate the groups of oyster samples from JZ, BJ, FG, GQ, JS and SY. One-way analysis of variance (ANOVA) was conducted on the PC scores from each group to test the statistical significance $(P<0.05)$ of separations. Furthermore, the supervised multivariate data analysis methods, partial least squares discriminant analysis (PLS-DA) and orthogonal projection to latent structure with discriminant analysis (O-PLS-DA), were sequentially carried out to uncover and extract the statistically significant metabolite variations related to metal pollutions. The results were visualized in terms of scores plots to show the classifications and corresponding loadings plots to show the NMR spectral variables contributing to the classifications. The model coefficients were calculated from the coefficients incorporating the weight of the variables in order to enhance interpretability of the model. Then metabolic differences responsible for the classifications between control (JZ) and metal pollution-exposed groups (JZ, BJ, FG, GQ, JS and SY) could be detected in the coefficient-coded loadings plots. The coefficient plots were generated by using MATLAB (V7.0, the Mathworks Inc., Natwick, USA) with an in-house developed program and were color-coded with absolute value of coefficients ( $r$ ). A hot color (i.e., red) corresponds to the metabolites with highly positive/negative significances in discriminating between groups, while a cool color (i.e., blue) corresponds to no significance. The correlation coefficient was determined on the basis of the test for the significance of the Pearson's product-moment correlation coefficient. The validation of the model was conducted using 10 -fold cross validation and the cross-validation parameter $Q^{2}$ was calculated, and an additional validation method, permutation test (permutation number $=200$ ), was also conducted in order to evaluate the validity of the PLS-DA models. The $\mathrm{R}^{2}$ in the permutated plot described how well the data fit the derived model, whereas $Q^{2}$ value describes the predictive ability of the constructed model and provides a measure of the model quality. If the maximum value of $Q^{2}$ max from the permutation test was smaller than or equal to the $Q^{2}$ of the real model, the model was regarded as a predictable model. Similarly, the $\mathrm{R}^{2}$ value and difference between the $\mathrm{R}^{2}$ and $Q^{2}$ were used to evaluate the possibility of over-fitted models (Feng et al., 2013). Metabolites were assigned following the tabulated chemical shifts and by using the software, Chenomx (Evaluation Version, Chenomx Inc., Edmonton, Alberta, Canada).

Each oyster tissue sample was dried to the constant weight and then digested in concentrated $\mathrm{HNO}_{3}$ at $80{ }^{\circ} \mathrm{C}$ for $12 \mathrm{~h}$, after which a clear liquid was obtained. Metal concentration in the oyster samples was determined by inductively coupled plasma mass spectrometry (ICP-MS, Agilent $7700 \times$ ). Appropriate internal standards ( $\mathrm{Sc}, \mathrm{Ge}$, In, Bi) were selected to correct the sensitivity drift and matrix effect. A quality control sample was repeatedly measured after every 10 samples. The recovery of the analyzed metals from the standard reference material (SRM $1566 \mathrm{~b}$, oyster tissue) was within $10 \%$ deviation from the certified values except $\mathrm{Cr}$, of which the certified concentration was not available. Metal concentrations were expressed as mean \pm standard deviation (S.D.). All the data of metal concentrations were subjected to principal component analysis with autoscaling and Student $t$-test. A P value less than 0.05 was considered significant. Statistical analysis was performed using Minitab software (Version 15, Minitab Inc. PA, USA).

As a hyperaccumulator of $\mathrm{Cu}$ and $\mathrm{Zn}$, oyster $\mathrm{C}$. sikamea can also accumulate high levels of other metals. The average concentrations ( $\mu \mathrm{g} / \mathrm{g}$ dry weight) of eight metals ( $\mathrm{Cr}, \mathrm{Ni}, \mathrm{Co}, \mathrm{Cu}, \mathrm{Zn}, \mathrm{Ag}, \mathrm{Cd}$ and $\mathrm{Pb}$ ) and one metalloid (As) in the oysters C. sikamea collected from clean (JZ) and metal contaminated sites (BJ, FG, GQ, JS and SY) are indicated in Table 1. To discern metal contamination situations, principal component analysis (PCA) were conducted to summarize the differences between different sampling sites, using the metal/metalloid concentrations as variables (Fig. 2). The biplot with samples (oyster samples from six sampling sites) and variables (eight metals and one metalloid) is shown in Fig. 2, with the first two principal components (PCs) containing $65.7 \%$ of the variance from the original nine dimensions. From the biplot with samples and variables, each variable's contribution to the first two components for clustering samples can be visualized directly. As shown in the biplot (Fig. 2), these six groups of samples from JZ, BJ, FG, GQ, JS and SY were clearly separated along both PC1 and/or PC2 axes, which demonstrated the significant differences on tissue metal concentrations between those samples from these six sampling sites. Overall, the oyster samples from BJ, FG and JS are clustered along positive PC1 axis with higher average tissue concentrations of $\mathrm{Cr}, \mathrm{Ni}, \mathrm{Co}$, $\mathrm{Cu}, \mathrm{Zn}, \mathrm{Ag}, \mathrm{Cd}$ and $\mathrm{Pb}$, while these oyster groups from JZ, GQ and SY are separated along negative PC1 axis. Especially, the oyster samples from JZ site accumulated a high level of As, since this metalloid is located near the sample cluster from JZ (Fig. 2). In details, the oyster samples from JS and $\mathrm{BJ}$ had the highest concentration of $\mathrm{Cu}$ and $\mathrm{Ag}$, respectively. In the biplot, both $\mathrm{Cd}$ and $\mathrm{Zn}$ are located in the cluster of oyster samples from FG, which indicated that these oyster samples were mainly contaminated by $\mathrm{Cd}$ and $\mathrm{Zn}$.

Simply to assess the severity of metal pollution in these six sampling sites, an equation suggested by Liu and Wang (2012) was used to evaluate the integrated metal contamination:

Integrated metal contamination $=\sum_{i=0}^{m} C_{\text {contaminated }}^{i}-C_{\text {clean }}^{i}$

where $C_{\text {contaminated }}^{i}$ is the concentration of the ith metal/metalloid in a contaminated oyster, and $C_{\text {clean }}^{i}$ is a reference value for the ith metal/ metalloid in an uncontaminated one. The reference was determined from the clean JZ oysters. And, $m$ is the number of all metals/metalloids that an organism was simultaneously exposed to, i.e., $m=9$ in this work. Although the oysters showed the highest tissue concentration of As, the integrated metal contamination suggested that JZ oysters were relatively clean compared with those oyster samples from other five sites. The integrated metal contamination values indicated that the oysters collected from JS, FG and BJ (JS > FG > BJ) sites were severely

Table 1

Metal/metalloid concentrations in the whole soft tissues from oysters C. sikamea sampled from six estuarine sites (JZ, BJ, FG, GQ JS and SY).

\begin{tabular}{|c|c|c|c|c|c|c|}
\hline \multirow{2}{*}{$\begin{array}{l}\text { Metal/metalloid concentration } \\
(\mu \mathrm{g} / \mathrm{g} \mathrm{dw})^{a}\end{array}$} & \multicolumn{6}{|l|}{ Sampling site } \\
\hline & $\mathrm{JZ}$ & $\mathrm{BJ}$ & FG & GQ & JS & SY \\
\hline $\mathrm{Cr}$ & $0.4 \pm 0.1$ & $10.3 \pm 1.8^{* *}$ & $13.4 \pm 2.7^{* *}$ & $13.1 \pm 2.6^{* *}$ & $12.2 \pm 2.4^{* *}$ & $1.5 \pm 0.6^{*}$ \\
\hline $\mathrm{Ni}$ & $3.1 \pm 0.2$ & $10.3 \pm 2.0^{* *}$ & $12.4 \pm 5.6^{* *}$ & $5.7 \pm 1.6^{*}$ & $10.0 \pm 2.1^{* *}$ & $3.1 \pm 1.1$ \\
\hline Co & $0.7 \pm 0.1$ & $1.6 \pm 0.3^{*}$ & $1.1 \pm 0.6$ & $0.8 \pm 0.1$ & $1.4 \pm 0.3^{*}$ & $0.8 \pm 0.2$ \\
\hline $\mathrm{Cu}$ & $406.2 \pm 77.1$ & $2963.7 \pm 757.8^{* *}$ & $2921.4 \pm 597.2^{* *}$ & $1413.7 \pm 570.5^{* *}$ & $6746.7 \pm 3331.9^{* *}$ & $744.1 \pm 256.3$ \\
\hline $\mathrm{Zn}$ & $4904.2 \pm 825.1$ & $5182.1 \pm 1922.0$ & $8991.5 \pm 1740.8^{*}$ & $4305.4 \pm 1769.7$ & $8409.8 \pm 2417.3^{*}$ & $4822.9 \pm 2328.2$ \\
\hline As & $30.6 \pm 4.8$ & $14.3 \pm 2.0^{* *}$ & $10.7 \pm 2.0^{* *}$ & $11.1 \pm 1.7^{* *}$ & $10.2 \pm 2.1^{* *}$ & $11.1 \pm 2.5^{* *}$ \\
\hline $\mathrm{Ag}$ & $3.1 \pm 0.3$ & $26.4 \pm 8.6^{* *}$ & $3.4 \pm 0.6$ & $2.7 \pm 1.6$ & $14.4 \pm 7.2^{* *}$ & $1.3 \pm 0.9^{*}$ \\
\hline $\mathrm{Cd}$ & $5.5 \pm 0.7$ & $7.6 \pm 0.9^{*}$ & $20.9 \pm 4.0^{* *}$ & $10.5 \pm 1.8^{* *}$ & $11.0 \pm 1.3^{* *}$ & $5.6 \pm 1.1$ \\
\hline $\mathrm{Pb}$ & $1.4 \pm 0.4$ & $4.9 \pm 0.6^{* *}$ & $3.4 \pm 0.3^{* *}$ & $4.4 \pm 1.6^{* *}$ & $4.9 \pm 1.6^{* *}$ & $3.2 \pm 1.7^{*}$ \\
\hline
\end{tabular}

a Data are shown as mean \pm standard deviation $(n=9$ or 10$)$. Values are presented as $\mu \mathrm{g} / \mathrm{g}$ dry weight.

* $(P<0.05)$ and $^{* *}(P<0.01)$ mean the significant differences of metal concentrations between clean (JZ) and metal pollution sites (BJ, FG, GQ JS or SY) (Student $t$-test). 


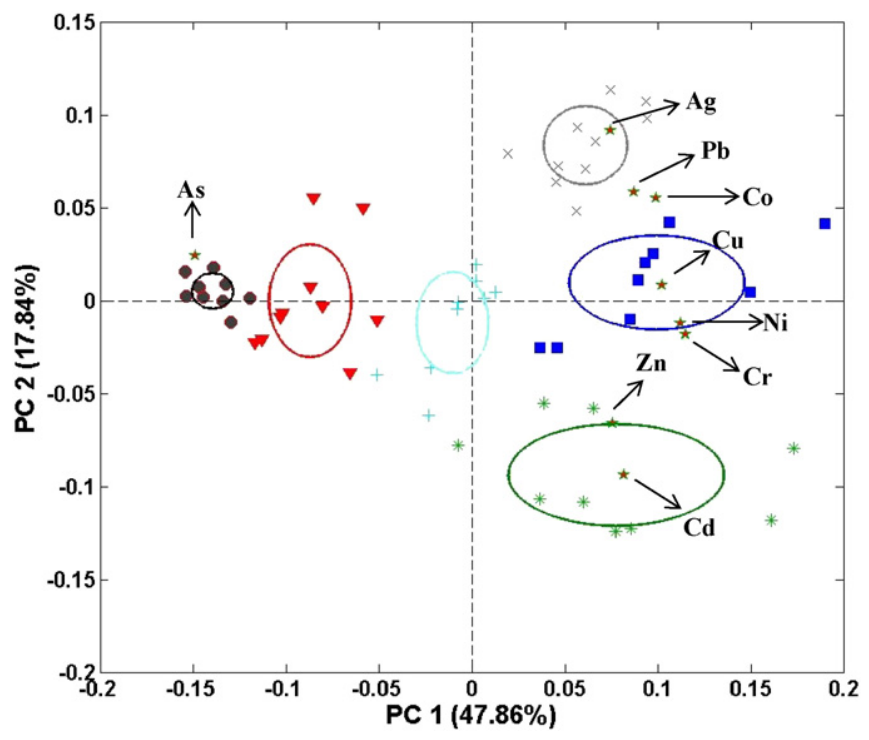

Fig. 2. The biplot containing PC scores of oyster samples from six sampling sites, Jiuzhen $(\mathrm{JZ}, \bullet$ ), Baijiao (BJ, $\times$ ), Fugong (FG, *), Gongqian (GQ +), Jinshan (JS, $\square$ ) and Songyu (SY, $\boldsymbol{\nabla}$ ) and variable (nine metals/metalloid) contributions for the clustering of oyster samples. Ellipses represent mean \pm standard deviation for each group of samples from different sites.

contaminated by the very high tissue concentrations of $\mathrm{Cu}$ and $\mathrm{Zn}$ (Table 1 and Fig. 2). In the biplot, the long distances of sample clusters from JS, FG and BJ to the sample cluster of JZ also confirmed that these three groups of oysters were heavily contaminated by metal pollution (Fig. 2). The integrated metal contamination value suggested oysters from SY were slightly contaminated compared with those samples from JZ samples, which was also demonstrated by the close clusters in the biplot (Fig. 2) and the similar levels of tissue metal concentrations (Table 1). As shown in Table 1, the relatively high tissue concentrations of $\mathrm{Pb}$ and $\mathrm{Cr}$ were observed in the SY samples. Although the oyster samples from GQ was not severely contaminated as those from JS, FG and BJ indicated by the integrated metal contamination, these oyster samples were still contaminated by the metals including $\mathrm{Cr}, \mathrm{Cu}, \mathrm{Cd}$ and $\mathrm{Pb}$.

A representative ${ }^{1} \mathrm{H}$ NMR spectrum of tissue extracts of oysters sampled from JZ is shown in both original (Fig. 3A) and glog-transformed (Fig. 3B) forms. Several different classes of metabolites were identified, including amino acids (valine, leucine, isoleucine, alanine, arginine, glutamate, glutamine, asparatate, glycine, etc.), osmolytes (betaine, taurine, homarine and hypotaurine), energy storage compounds (glucose, glycogen and ATP) and intermediates in the Krebs cycle (succinate and fumurate). Visibly, all of the ${ }^{1} \mathrm{H}$ NMR spectra were dominated by organic osmolytes, such as betaine and taurine, which are used to regulate the osmolarity between oyster and seawater (Fig. 3).

PCA was conducted on the ${ }^{1} \mathrm{H}$ NMR spectral datasets to summarize the similarities and differences between different sample groups. The PC scores plot presented significant $(P<0.01)$ separations between clean (JZ) and metal pollution-exposed groups (BJ, FG, GQ JS and SY), along both PC1 (51.72\% of variation) and PC2 (15.98\% of variation) axes (Fig. 4), which suggested that there were significant metabolic responses in these five groups of oysters exposed to metal pollutions. Furthermore, O-PLS-DA was performed on the ${ }^{1} \mathrm{H}$ NMR spectral datasets from control and metal pollution-exposed groups, respectively (Fig. 5). The scores plots (Fig. 5A, C, E, G and I) derived from O-PLS-DA indicated clear classifications between control (JZ) and metal pollution-exposed groups (BJ, FG, GQ JS and SY), respectively, with reliable $Q^{2}$ values $(>0.9)$. The significant metabolic responses related to metal pollutions of these sampling sites were labeled in the corresponding loading plots of O-PLS-DA (Fig. 5B, D, F, H and J).

Since the six investigated sampling sites were of similar water physico-chemical parameters (i.e. temperature, salinity, $\mathrm{pH}$, dissolved

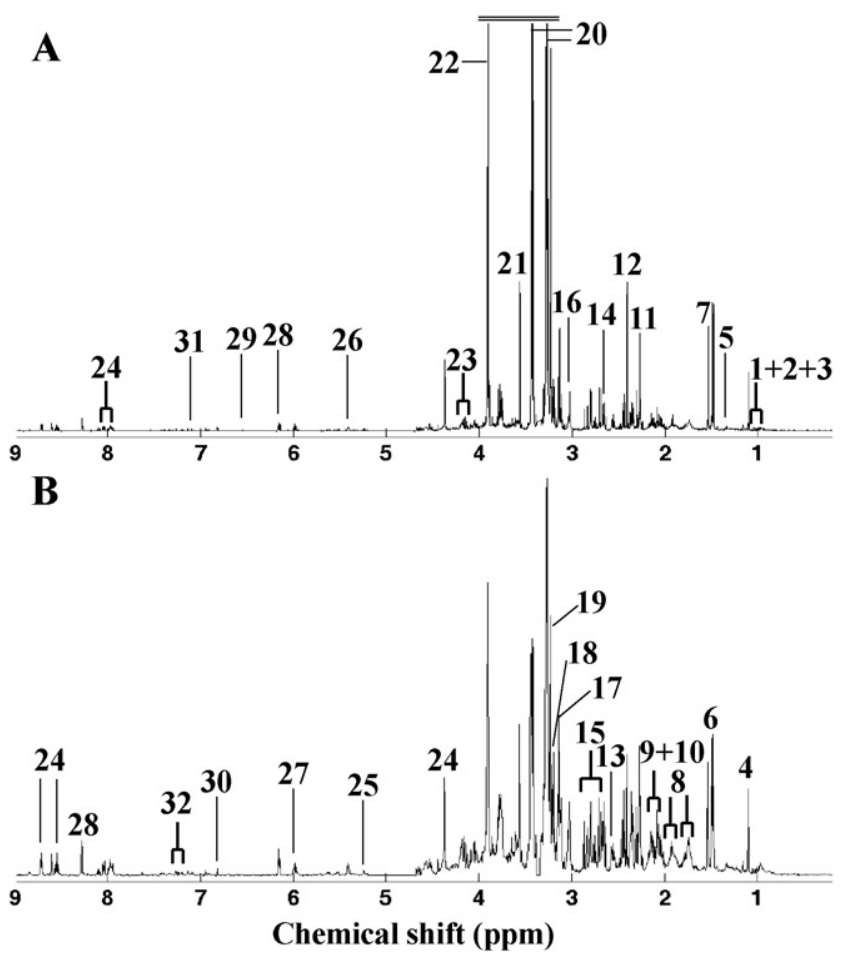

Fig. 3. One representative 1-dimensional $500 \mathrm{MHz}{ }^{1} \mathrm{H}$ NMR spectrum of the whole tissue extracts from oysters C. sikamea from the clean site, Jiuzhen in original (A) and generalized log transformed (B) forms. Keys: (1) leucine, (2) isoleucine, (3) valine, (4) unknown 1 (1.10 ppm), (5) threonine, (6) alanine, (7) unknown 3 (1.50 ppm), (8) arginine, (9) glutamate, (10) glutamine, (11) acetoacetate, (12) succinate, (13) $\beta$-alanine, (14) hypotaurine, (15) aspartate, (16) lysine, (17) malonate, (18) choline, (19) phosphocholine, (20) taurine, (21) glycine, (22) betaine, (23) proline, (24) homarine, (25) glucose, (26) glycogen, (27) unknown 3 (5.96 ppm), (28) ATP, (29) fumarate, (30) tyrosine, (31) histidine and (32) phenylalanine.

oxygen) (Liu and Wang, 2012), the differential metabolic profiles should be related to the different metal pollutions in the sampling sites. Firstly, we characterized the metabolic responses in the oysters from these three heavily polluted sites, BJ, FG and JS. As shown in the

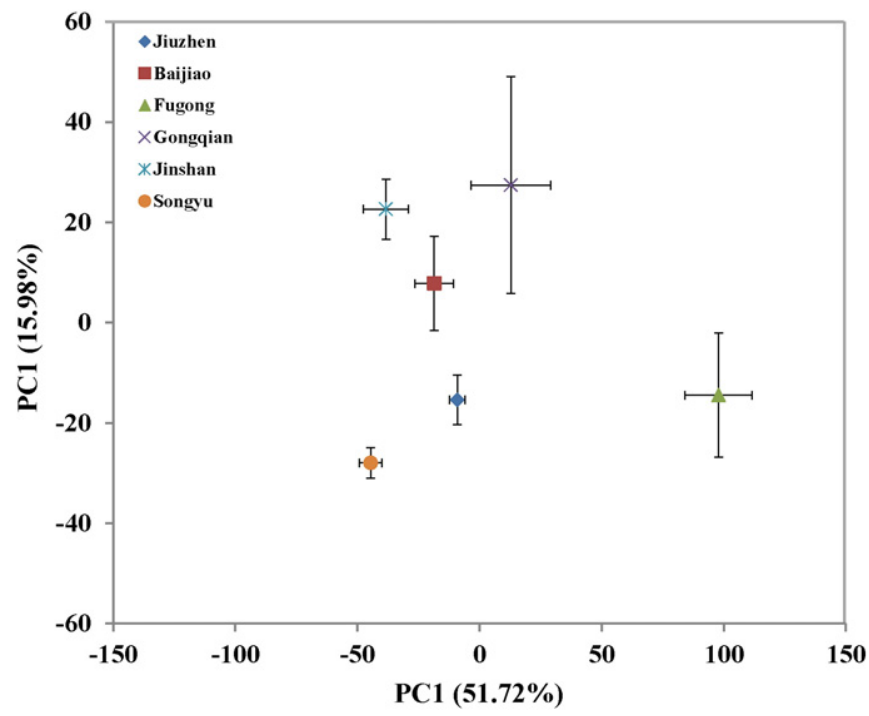

Fig. 4. Mean principal component analysis (PCA) scores plot of PC1 versus $\mathrm{PC} 2$ for ${ }^{1} \mathrm{H}$ NMR spectra of the whole tissue extracts from oysters $C$. sikamea sampled from Jiuzhen $(\diamond)$ Baijiao ( $\bullet$ ), Fugong $(\Delta)$, Gongqian $(\times)$, Jinshan $(*)$ and Songyu $(\bullet)$ sites. The classes were presented as mean \pm standard deviation (as associated standard error) of PC scores for each group of oyster samples. 

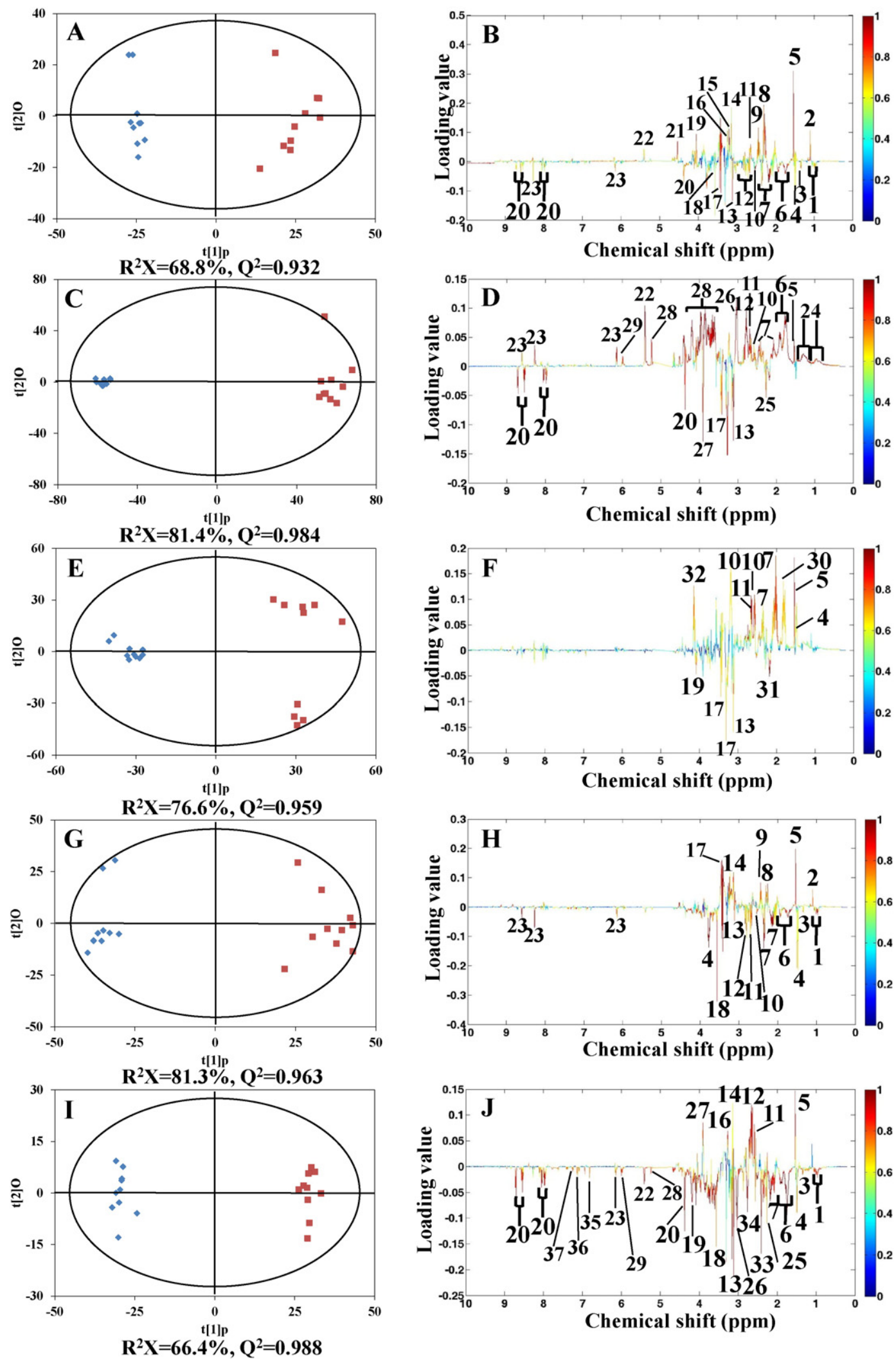

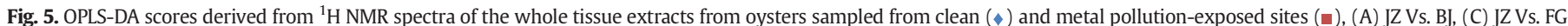

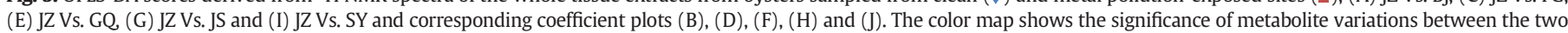

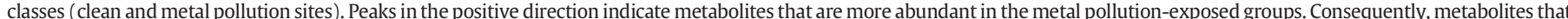

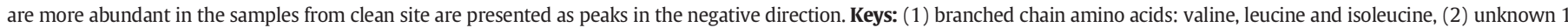

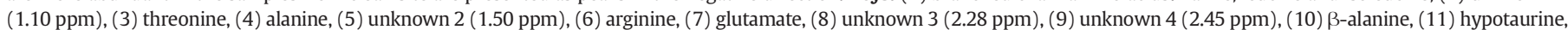

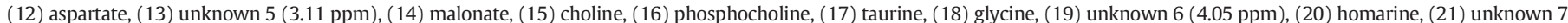

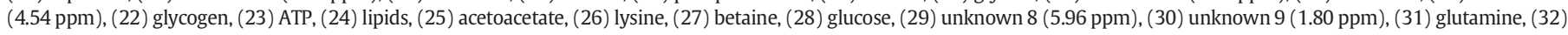
proline, (33) succinate, (34) unknown 10 (2.77 ppm), (35) tyrosine, (36) histidine and (37) phenylalanine. 
loading plot of O-PLS-DA (Fig. 5B), the oyster samples from BJ had higher concentrations of hypotaurine, malonate, choline, phosphocholine and glycogen and lower concentrations of branched chain amino acids (valine, leucine and isoleucine), threonine, alanine, arginine, glutamate, $\beta$-alanine, aspartate, taurine, glycine, homarine and ATP, than those in the oyster samples from the clean site, JZ estuary. Compared with those oyster samples from control site (JZ), the metabolic profiles in the oyster samples from FG site presented higher levels of alanine, arginine, glutamate, $\beta$-alanine, hypotaurine, aspartate, glycogen, ATP, lysine and glucose and lower levels of taurine, homarine, acetoacetate and betaine (Fig. 5D). For the oyster samples from JS site, malonate and taurine were elevated and BCAAs, threonine, alanine, arginine, glutamate, $\beta$-alanine, hypotaurine, aspartate, glycine and ATP were depleted.

The metabolic responses including malonate, BCAAs, threonine, alanine, arginine, glutamate, $\beta$-alanine, aspartate, glycine and ATP were similarly altered in the oysters from BJ and JS. However, the metabolic profile of oysters from FG was quite different compared with these from BJ and JS. Since all the oysters from BJ, FG and JS were severely contaminated by both $\mathrm{Cu}$ and $\mathrm{Zn}$ as mentioned above, the differential metabolic responses in oysters from BJ and JS sites compared with those from FG sites might be caused by other accumulated metal(s). Interestingly, as observed in Table 1, the tissues concentrations of Ag in the oysters from both BJ and JS sites were much higher than that in the oyster samples from FG. It seemed that the accumulated Ag induced significant effects in the oysters from both BJ and JS sites. Free amino acids in marine mollusks are used as osmolytes to balance their intracellular osmolarity with the environment (Viant et al., 2003; Kwon et al., 2012). In another pathway, amino acids can be oxidized to supply energy in Krebs cycle. Malonate is a competitive inhibitor of the enzyme succinate dehydrogenase by binding to the active site of the enzyme without reacting. In the oysters from both BJ and JS sites, the amino acids including BCAAs, threonine, alanine, arginine, glutamate, $\beta$-alanine, aspartate and glycine were significantly decreased, whereas ATP and malonate were increased. These metabolic responses suggested that metal pollutions probably affected the Krebs cycle related to energy metabolism in oysters from BJ and JS sites. Interestingly, the two known organic osmolytes, taurine and hypotaurine, were contrarily altered in the oysters from BJ and JS sites, which implied that the metal pollutions induced obvious osmotic stress in oysters via different pathways. As shown in Table 1, the tissue concentrations of $\mathrm{Cu}$ and $\mathrm{Zn}$ in the oysters from JS site are almost 2 times higher than those from BJ site. In a previous study, Zn exposure induced increased taurine and decreased hypotaurine in white clam Ruditapes philippinarum, which was similar to the altered taurine and hypotaurine in oysters from JS site (Wu et al., 2013). Therefore, the contrarily alterations of hypotaurine and taurine in oysters from JS site might be related to the higher accumulation of $\mathrm{Zn}$. Choline and phosphocholine are involved in a reaction catalyzed by choline kinase converting ATP and choline into phosphocholine and ADP. In the oysters from BJ, choline and phosphocholine were significantly elevated, which suggested that this reaction was enhanced in oysters. Li et al. (2015) reported that the phosphocholine was also elevated in $\mathrm{Ag}^{+}$-exposed Eisenia fetida. Since the oysters from $\mathrm{BJ}$ site accumulated the highest concentration of $\mathrm{Ag}$, the elevation of phosphocholine might be the metabolic biomarker of Ag. The increased glycogen implied the disturbance in energy metabolism in oysters from BJ site, which is another differential response compared with that in oysters from JS site.

The increased amino acids including alanine, arginine, glutamate, $\beta$-alanine, aspartate and lysine indicated the osmotic stress induced by the metal pollution in the oysters from FG site, together with the increased osmolyte, hypotaurine (Fig. 5D). However, other three osmolytes including taurine, homarine and betaine were significantly decreased. These decreased osmolytes were probably used to compensate for the increased amino acids and hypotaurine in the oysters from FG site. The elevated glycogen, glucose and ATP might mean the enhanced energy demand in oysters from FG site caused by the metal pollution. Acetoacetate is a ketone body and can be synthesized from acetyl-coenzyme A (acetyl-CoA) as the end product of fatty acid metabolism. The low concentrations of acetoacetate implied the reduced lipid metabolism in oysters from FG site.

Although the oysters from GQ and SY sites were less severely contaminated by metals/metalloid, the significant metabolic responses were detected (Fig. 5F and J). Basically, the amino acids including alanine, glutamate, $\beta$-alanine and proline were significantly elevated induced by metal pollution together with the osmolyte, hypotaurine in oysters from GQ site. Additionally, taurine, glutamine and other unknown metabolites ( 3.11 and $4.05 \mathrm{ppm}$ ) were depleted. Obviously, the elevated amino acids and hypotaurine indicated the osmotic stress in oysters from GQ site, whereas the depleted taurine and glutamine were used to compensate for the increased amino acids and hypotaurine.

For the oysters from SY sites, the main metal contaminants included $\mathrm{Cr}$ and $\mathrm{Pb}$ (Table 1). The metal pollution induced metabolic responses included the increased hypotaurine, aspartate, malonate, phosphocholine and betaine, and decreased BCAAs, threonine, alanine, arginine, glycine, homarine, glycogen, ATP, glucose, succinate, tyrosine, histidine and phenylalanine. The altered osmolytes, betaine and homarine, and amino acids indicated the disturbance in osmotic stress induced by metal pollution in oysters from SY site. As mentioned above, malonate is a competitive inhibitor of the enzyme succinate dehydrogenase. Therefore, the increased malonate might result in decreased succinate that is involved in Krebs cycle and was observed in the oysters from SY site. Phosphocholine can be synthesized in the reaction catalyzed by choline kinase resulting in reduced ATP and choline. Therefore, the increased phosphocholine and decreased ATP implied the reduced energy metabolism caused by metal pollution in oysters from SY, which was also evidenced by the decreased glycogen and glucose. In details, alanine constitutes the major portion of end-product of glucose breakdown anaerobically, together with the metabolite of succinate in invertebrate (Stokes and Awapara, 1968). In addition, there could be a substantial conversion of aspartate to succinate under anoxic conditions in mollusk hence with decreased aspartate and increased succinate (Graham and Ellington, 1985). Interestingly, increased aspartate and decreased succinate and alanine were found in the oysters from SY site. Therefore, the high levels of aspartate and low levels of alanine and succinate demonstrated the reduced anaerobiosis induced by metal pollution in oysters from FG site.

As indicated by the tissue concentrations of metals/metalloid, the oyster samples from JS and BJ had the highest concentration of $\mathrm{Cu}$ and Ag, respectively. The oysters from FG site were mainly contaminated by $\mathrm{Cd}$ and Zn. Although the oysters from GQ and SY sites were less severely contaminated by metals/metalloid, the significant accumulated metals such as $\mathrm{Cr}, \mathrm{Cd}$ and $\mathrm{Pb}$ were detected. In this work, the metabolic differences between oysters $C$. sikamea from clean (Jiuzhen) and metal pollution sites (Baijiao, Fugong, Gongqian, Jinshan and Songyu) were characterized using NMR-based metabolomics. Basically, the metal pollutions in BJ, FG, JS and SY sites induced disturbances in osmotic regulation and energy metabolism via different metabolic pathways indicated by the different metabolic biomarkers compared with those oyster samples from the clean site, JZ. However, the metal pollution in GQ site mainly influenced the osmotic regulation in the oysters $C$. sikamea. This study demonstrates that NMR-based metabolomics is a useful to characterize metabolic responses induced by metal pollution.

\section{Acknowledgments}

This work was supported by a Key Project from NSFC (21237004) and National Key Basic Research Program of China (2015CB453303). We thank Prof. Mark Viant for the use of ProMetab software. 


\section{References}

Cappello, T., Maisano, M., D'Agata, A., Natalotto, A., Mauceri, A., Fasulo, S., 2013a. Effects of environmental pollution in caged mussels (Mytilus galloprovincialis). Mar. Environ. Res. 91, 52-60.

Cappello, T., Mauceri, A., Corsaro, C., Maisano, M., Parrino, V., Lo Paro, G., Messina, G., Fasulo, S., 2013b. Impact of environmental pollution on caged mussels Mytilus galloprovincialis using NMR-based metabolomics. Mar. Pollut. Bull. 77, 132-139.

de Almeida, E.A., Miyamoto, S., Bainy, A.C.D., de Medeiros, M.H.G., Mascio, P., 2004. Protective effect of phospholipid hydroperoxide glutathione peroxidase (PHGPX) against lipid peroxidation in mussels Perna perna exposed to different metals. Mar. Pollut. Bull. 49, 386-392.

Fasulo, S., Iacono, F., Cappello, T., Corsaro, C., Maisano, M., D'Agata, A., Giannetto, A., de Domenico, E., Parrino, V., Lo Paro, G., Mauceri, A., 2012. Metabolomic investigation of Mytilus galloprovincialis (Lamarck 1819) Caged in aquatic environments. Ecotoxicol. Environ. Saf. 84, 139-146.

Feng, J., Li, J., Wu, H., Chen, Z., 2013. Metabolic responses of HeLa cells to silica nanoparticles by NMR-based metabolomic analyses. Metabolomics 9, 874-886.

Goldberg, E.D., Koide, M., Hodge, V., Flegal, A.R., Martin, J., 1983. United States mussel watch - 1977-1978 results on trace metals and radionuclides. Estuar. Coast. Shelf Sci. 16, 69-93.

Graham, R.A., Ellington, W.R., 1985. Anaerobic aspartate metabolism and the formation of alanine in molluscan cardiac muscle: A ${ }^{13}$ C NMR study. J. Exp. Zool. 236, 365-370.

Hines, A., Oladiran, G.S., Bignell, J.P., Stentiford, G.D., Viant, M.R., 2007. Direct sampling of organisms from the field and knowledge of their phenotype: key recommendations for environmental metabolomics. Environ. Sci. Technol. 41, 3375-3381.

Ji, C., Wu, H., Wei, L., Zhao, J., Yu, J., 2013. Proteomic and metabolomic analysis reveal gender-specific responses of mussel Mytilus galloprovincialis to 2,2',4,4'tetrabromodiphenyl ether (BDE 47). Aquat. Toxicol. 140-141, 449-457.

Jones, O.A.H., Dondero, F., Viarengo, A., Griffin, J.L., 2008. Metabolic profiling of Mytilus galloprovincialis and its potential applications for pollution assessment. Mar. Ecol. Prog. Ser. 369, 169-179.

Kim, H.K., Choi, Y.H., Verpoorte, R., 2010. NMR-based metabolomic analysis of plants. Nat. Protoc. 5, 536-549.

Kwon, Y.K., Jung, Y.S., Park, J.C., Seo, J., Choi, M.S., Hwang, G.S., 2012. Characterizing the effect of heavy metal contamination on marine mussels using metabolomics. Mar. Pollut. Bull. 64, 1874-1879.

Li, L., Wu, H., Peijnenburg, W.J.G.M., van Gestel, C.A.M., 2015. Both released silver ions and particulate Ag contribute to the toxicity of AgNPs to earthworm Eisenia fetida. Nanotoxicology. http://dx.doi.org/10.3109/17435390.2014.976851.

Liu, F., Wang, W.-X., 2012. Proteome pattern in oysters as a diagnostic tool for metal pollution. J. Hazard. Mater. 239-240, 241-248.
Rank, J., Lehtonen, K.K., Strand, J., Laursen, M., 2007. DNA damage, acetylcholinesterase activity and lysosomal stability in native and transplanted mussels (Mytilus edulis) in areas close to coastal chemical dumping sites in Denmark. Aquat. Toxicol. 84 $50-61$.

Regoli, F., 2000. Total oxyradical scavenging capacity (TOSC) in polluted and translocated mussels: a predictive biomarker of oxidative stress. Aquat. Toxicol. 50, 351-361.

Santos, E., Ball, J.S., Williams, T.D., Wu, H., Ortega, F., Van Aerle, R., Katsiadaki, I., Falciani, F. Viant, M.R., Chipman, J.K., Tyler, C.R., 2010. Identifying health impacts of exposure to copper using transcriptomics and metabolomics in a fish model. Environ. Sci. Technol. $44,820-826$.

Stokes, T.M., Awapara, A., 1968. Alanine and succinate as end-products of glucose degradation in the clam Rangia cuneata. Comp. Biochem. Physiol. 25, 883-892.

Tan, Q.-G., Wang, Y., Wang, W.-X., 2015. Speciation of Cu and Zn in two colored oyster species determined by X-ray absorption spectroscopy. Environ. Sci. Technol. 49 6919-6925.

Tarachiwin, L., Masako, O., Fukusaki, E., 2008. Quality evaluation and prediction of Citrullus lanatus by ${ }^{1} \mathrm{H}$ NMR-based metabolomics and multivariate analysis. J. Agric Food Chem. 56, 5827-5835.

Wang, W.-X., Pan, K., Tan, Q., Luo, L., Simpson, S.L., 2014. Estuarine pollution of metals in China: science and mitigation. Environ. Sci. Technol. 48, 9975-9976.

Weng, N., Wang, W.-X., 2014. Variations of trace metals in two estuarine environments with contrasting pollution histories. Sci. Total Environ. 485-486, 604-614.

Williams, T.D., Wu, H., Santos, E., Ball, J., Katsiadaki, I., Brown, M.M., Baker, P., Ortega, F., Falciani, F., Craft, J.A., Tyler, C.R., Chipman, J.K., Viant, M.R., 2009. Hepatic transcriptomic and metabolomic responses in the stickleback (Gasterosteus aculeatus) exposed to environmentally relevant concentrations of dibenzanthracene. Environ. Sci. Technol. 43, 6341-6348.

Wu, H., Ji, C., Wang, Q., Liu, X., Zhao, J., Feng, J., 2013. Manila clam Venerupis philippinarum as a biomonitor to metal pollution. Chin. J. Oceanol. Limnol. 31, 65-74

Viant, M.R., Rosenblum, E.S., Tjeerdema, R.S., 2003. NMR-based metabolomics: a powerful approach for characterizing the effects of environmental stressors on organism health. Environ. Sci. Technol. 37, 4982-4989.

Vidal-Liñán, L., Bellas, J., 2013. Practical procedures for selected biomarkers in mussels Mytilus galloprovincialis Implications for marine pollution monitoring. Sci. Total Environ. 461-462, 56-64.

Zhang L, Liu, X, You, L, Zhou, D, Wang Q, Li, F. Cong M., Li, L, Zhao, J. Liu, D, Yu, J, Wu, H., 2011. Benzo(a)pyrene-induced metabolic responses in Manila clam Ruditapes philippinarum by proton nuclear magnetic resonance ( ${ }^{1} \mathrm{H}$ NMR) based metabolomics. Environ. Toxicol. Pharmacol. 32, 218-225. 